\title{
Complex integrative etiological (possibly encephalomyelitis), clinical, paraclinic and therapeutic aspects in a patient with quite light spastic paraparesis and sensitivity disorders, old objected by medullar mitigation at vertebral level T5-T7 - Case report
}

\author{
Irina Raluca Petcu ${ }^{2}$,Mihaela Mandu², Nicoleta Chiriloi ${ }^{2}$, Anca Chiralidis ${ }^{2}$, Mihai Băilă², Doroteea \\ Teoibaș-Șerban $^{1,2}$, Simona Izabelle Stoica ${ }^{1,2}$, Gelu Onose ${ }^{1,2}$
}

Corresponding author: Irina Raluca PETCU, E-mail address: varsa_raluca@yahoo.com

1 The Teaching Emergency Hospital "Bagdasar-Arseni” (TEHBA), Bucharest, Romania 2 The University of Medicine and Pharmacy”Carol Davila” (UMPCD), Bucharest, Romania

\begin{abstract}
Introduction: This paper presents an extremely complex case of quite light spastic paraparesis, with a medical history from childhood (at 12 years), encephalomyelitis (remission?), which in adulthood (at 22 years) was diagnosed and treated as multiple sclerosis 1 year and 6 months (this diagnosis has been subsegmently deniend with IRM in 2017: reducing the size of the medullary cord in the vertebrae plane T5-T7 sequelae aspect) and the afferent neuro-rehabilitative actual management approach, respectively.

Materials and methods: 43 year old female patient admintted in our Clinic s Division for a AIS/ Frankel D motor deficiency, pain with mechanical carcass at the spine, disturbances of balance, bladder dysfunction (incontinance), dorsal plantar flexion deficit bilateral (left > right), gait with a broad base of support with external unilateral support, with hip flexural , knee flexion, and dorsal flexion deficiency while walking, attack digitigrad (left > right), and sensibility impairment from T12 level downwards. The patient was clinical and functionally evaluated, according to the standard implemented protocols of our Unit , through the following measurament evalution scales: AIS, Functional Independence Measure (FIM), QQL (Quality of life), Ashworth, Penn, FAC International Scale, and investigate paraclinic: IRM brain, cervical and thoracic spine, radiographic cervical spine , abdominal ecography.

Results: Following optimal treatment including pharmacological, and complex neuro-rehabilition program the patient had a favorable evolution with increased values of the measurament scales (motor AIS with 4 points, FIM motor with 5 points, QQL with 6 points, and FAC with 2 points) ), remission of ataxic / vertiginous phenomena, quaremision of urinary incontinence (controlling micturitions for at least 10 minutes after urge to urinate); in addition and also very important: diminished spasticity and significantly improved of the gait pattern.Now shie can walk without support on short / medium distances and also very important it can rise without sitting support (in a relatively low position) to orthostatism, and also climbs and descends stairs with the support of the bar and only with the supervision of another person.

Conclusion: From the etiologic point of view, multiple sclerosis, uterine apoplexy as well arterio-venous malformation has been recently refuted. The present case represents the importance of building a complete diagnosis (etiologic and of stage) and particular neuro-rehabilitative therapy approuch with both clinical, psihological and scientific impact.
\end{abstract}

Key words: spastic paraparesis, neuro-rehabilitative therapy

\section{Introduction}

Encephalomyelitis is both an inflammatory disorder of the encephalus and of the spinal cord. Concomitant signs of encephalitis (somnolence, behavioral disorders, convulsions, etc.), myelitis (paralysis with sensorial-sensory changes below this level) and meningitis (neck revascularisation, headache and fever) occur. In $60-70 \%$ of cases, encephalomyelitis is preceded by a viral infection. The most common viruses are measles virus, varicella-zoster virus, rubella influenza and paragripal viruses, Ebstein Barr virus.

Despite the progress that has been made with both classical virological and molecular biological technologies in identifying the numerous causes of encephalomyelitis, there is an increasing need for better tools and new approaches for detecting both genuinely novel pathogens and determining new manifestations of known pathogens, including viruses. In evaluating the results of different studies, it is crucial to have uniform clinical and diagnostic criteria for establishing a definite diagnosis of encephalomyelitis. There is not a standardized algorithm for the molecular investigation of unexplained encephalomyelitis, though general guidelines for the investigation and management of suspected encephalomyelitis are now available.

The prognosis is dictated by the pathogenic agent's virulence and pre-existing health condition. Extreme ages, the state of immunodepression, preexisting neurological disorder are associated with worse prognosis.

Untreated, mortality in herpes encephalitis reaches $50-75 \%$, and those who survive remain with neurological sequelae and various disabilities. In the treated one, the mortality is $20 \%$ and the severity of the sequelae is influenced by the duration of the time span from onset to initiation of treatment. 


\section{MATERIALS AND METHODS:}

This paper presents the case of a patient having The Teaching Emergency Hospital "Bagdasar-Arseni", TEHBA, Bioethics Committee approval no 9181/11.04.2018.

A 43 year old female patient admintted in our Clinic s Division for: lower limb paralysis motor deficiency (predominantly inferior stg.), bladder dysfunction (incontinance), ataxic/ vertigo positional syndrome, pain with mechanical character in the cervical, thoracic and lumbar spine, sensitivity disorders at the lower limb with T12 neurological level.

From the personal pathologic history we keep the following: Encephalitis episode in 1986 ( clinic :neck blunt, high fever, headache, vomiting, disturbances of balance; paraclinic: LCR-clear, EEG-discrete diffuse bioelectric changes, skull radiography normal and the treatment was antiepileptics, antithermal , antibiotics with good evolution) and Multiple sclerosis in 1996 (clinic: pain in the bilateral calcanean region with an ascendent evolution, at 3 months postpartum the spastic paraparesis and sphincter disorders take place and in 2001- right trigeminal neuralgia; paraclinic: MRI cerebral normal and the treatment was ACTH-10 days after that with Prednison -affirmative ; Interferon in 2002 for 1 year and 6 months).

Female patient, with a chidhood medical history (at the age of 12 ) encephalomyelitis who was diagnosed in adulhood (at the age of 22 ) was diagnosed with multiple sclerosis on clinical criteria, for which she had an immunosuppressive treatment for 1 year and 6 months, she's being hospitalized for quite light spastic paraparesis, disorder of gait and balance aggravated in the last 6 months, ataxic/ vertigo positional syndrome, bladder dysfunction (incontinance), sensitivity disorders at the lower limb and pain with mechanical character in the cervical, thoracic and lumbar spine.

Examination objective upon admission: good general condition, afebrile, normoponderal, normal in teguments and mucosae, superficial non-palpable lymph nodes; respiratory system: normal vesicular breathing, without patological bronchial sounds, $\mathrm{SaO} 2=98 \%$ spontaneous; cardiovascular system: normal heart sound, no added sounds or murmer, $\mathrm{BP}=120 / 80 \mathrm{mmHg}$, heart rate $73 \mathrm{bpm}$ rhythmic, the pedios pulsatile bilateral artery; digestive system: slim abdomen, mobil with breathing, painlessly spontaneos and palpation, slow intestinal tranzit (approximate 5 days) helped with parafine oil, liver with inferior margin at the coastal rebord, nonpalpable spleen; urogenital system: Giordano negative, bladder dysfunction (incontinance); muscular system: hypertonia lower limb bilateral, left> right; osteo-articular system: right coxalgia, idiopathic scoliosis thoraco-lumbar, pain with mechanical character in the cervical, thoracic and lumbar spine, straightening of the cervical spine.

NMAK Examination: Patient conscious, cooperative, spatio-temporal orientation and allopsychic, paraparetic motor deficitin lower limbs (left > right), lower limb motor score: 34/50 ( left $16 / 25$, right $18 / 25$ ), upper limbs: 50/50, FIM cognitive subtotal score 35/35; QOL $=108 / 112$; superficial sensitivity disorders at the lower limb with T12 neurological level.

Babinski is present bilaterally; reflexes osteodentinous: live in the lower limbs; ataxic/ vertigo positional syndrome, Romberg positive, horizontal nystagmus present at the sudden change of position (from standstill and vice versa); spasticity in bilateral lower limbs: : score Asworth 2 at the level of the left lower limb and $1+$ at the level of the right lower limb; bilateral ankle clonus epuizable from 510 secuse.

Functional, the patient moves very hard with unilateral support, shows walking with broad support base, hip flexural deficit, knee flexion, and dorsal flexion, digitigrade attack (left lower limb> right lower limb), disturbance of balance.

Paraclinically-imaging:

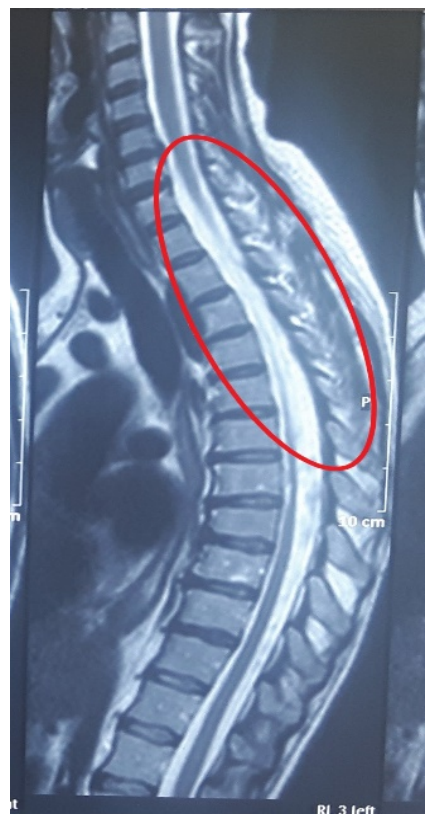

(Fig1.) Cervico-toracal native MRI it shows the reduction in the size of the medullary cord in the vertebrae plane T5-T7 (sechelar aspect) but without signal changes at its level, no spinal space replacement processes.

(Fig.1) 

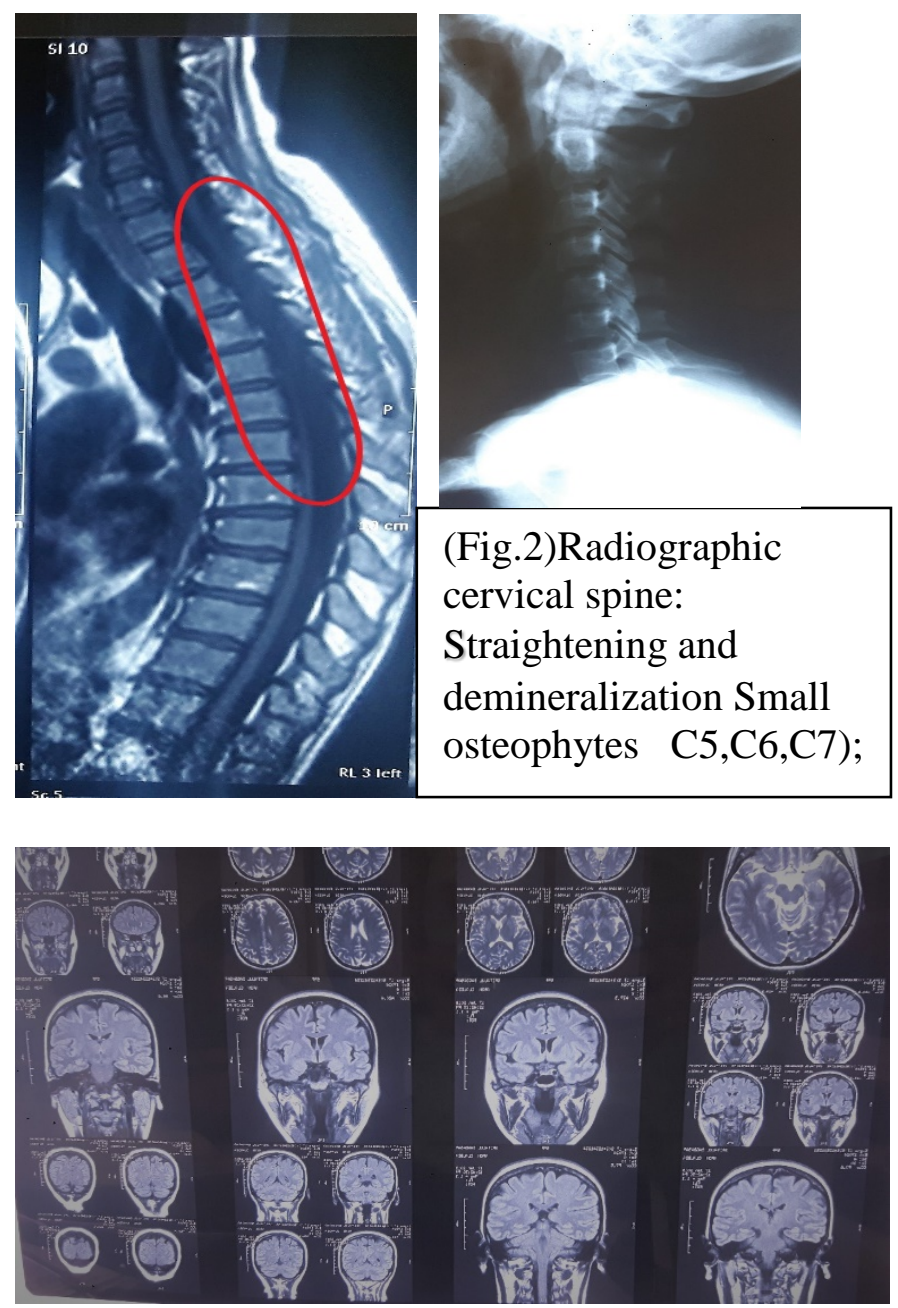

(Fig.3) Brain nativ MRI : No pathological cerebral and signal changes

The patient performed MRI thoracic angiography in 2017, where the possibility of vascular malformation was denied.

Tratament: During the hospital stay, the patient underwent a complex recovery program which included: treatment with medication (central antispasmodic, urinary antiseptics, anticholinergic , antivertigo agents, bone antiresorbent, antiinflammatory gel applied locally when needed), with tolerability and good evolution; physical treatment (kinesiotherapy at gym).

RESULTS: Having a complex neuromuscular recovery program, the patient had a favorable evolution with increasing the scale values (AIS motor with 4 points, FIM motor with 5 points, QQL with 6 points, and FAC with 2 points), remission of ataxic / vertiginous phenomena, quaremision of urinary incontinence (controlling micturitions for at least 10 minutes after urge to urinate); in addition and also very important: diminished so it can be raised without sitting support (in a relatively low position). The gait pattern has significantly improved: performing hip flexion and planting dorsiflexion in the balancing phase, increasing gait speed, synchronizing movements of the torso and upper limb with the lower limbs, awareness of gait phase, the patient can go without support on short / medium distances.

The patient was discharged from our clinic with the following appointments: continuation of the recovery program according to the instructions given at discharge; regular check-up at our clinic; hydrokinetherapy in units with neuro-rehabilitation experience, (Techirghiol in May, June, September, eventually Felix).

We note and point out that the patient, although old in terms of neurological suffering, at our first admission and the native cerebral MRI did not reveal brain damage. It is to be noted that the patient due to the very old suffering that has not achieved a significant clinico-functional improvement search for diagnosis and consistent therapeutic recovery results including abroad (Viena, Privatklinik Döbling).

Afterwards, as it can be observed, the patient did not get the desired results, this being the reason why she addressed and was hospitalized in our Clinic.

CONCLUSION: From an etiological point of view, multiple sclerosis, uterine apoplexy as well as arterio-venous malformation have been recently refuted. The present case shows the importance of building a complete diagnosis (etiological and of stage) and particular neuro-rehabilitative therapy approach with not only clinical, but also psychological and scientific beneficial impact.

\section{Bibliography}

1. Onose G., Padure L., "Compendiu de Neuroreabilitare - la adulţi, copii şi vârstnici Editura Universitara "Carol Davila", Bucuresti, 2008.

2. https://www.ninds.nih.gov/Disorders/All-Disorders/AcuteDisseminated-Encephalomyelitis-Information-Page

3. http://www.msdmanuals.com/professional/neurologicdisorders/brain-infections/encephalitis

4. https://www.medicalnewstoday.com/articles/168997.php

5. https://www.ncbi.nlm.nih.gov/pmc/articles/PMC5490815/

6. http://www.csid.ro/boli-afectiuni/neurologie/encefalita13501793 